\title{
The Study on the Dynamic Multi-objective Recognition and Estimation Algorithm of Infrared Imaging Based on Particle Swarms Collaboration
}

\author{
Lang Zhai* and Qi Hu
}

\author{
College of Information Engineering, Jilin Business and Technology College, Changchun, Jilin, 130062, China
}

\begin{abstract}
Recently, a problem that the infrared decoy interferes infrared detection system, cannot be solved. With the gradual application and popularity of the particle swarm optimization, it is preferable to apply it to dynamic multiobjective optimization to solve the problem of the recognition and the estimation of dynamic multi-object in infrared imaging. In this study, the dynamic multi-objective estimation and recognition algorithm of the infrared imaging, which is based on the multi-particle swarms collaboration, ultimately estimates the motion trajectory and Pareto optimal solution of the infrared imaging through the continuous improvement and upgradation of the particle swarms optimized algorithm, the continuous study and inheritance as well as the combination with the aerodynamic characteristic of the infrared decoy. The experiment proves that the improvement of particle swarm algorithm efficiently reduces the estimation error, which produces favorable optimized effects. The experiment has great engineering significance.
\end{abstract}

Keywords: DMOP, Infrared imaging 1, Multiple particle swarms, Pareto optimal solutions.

\section{INTRODUCTION}

The infrared imaging technology has advantages in target detection, recognition and anti-interference, thus it gradually attracts a wider attention. As the imaging technology develops, new requirements are put forward for the test and the estimation of imaging system and its corresponding infrared detection weapon technology. As for the infrared imaging sensor, its main function is to trace and recognize the tail flame imaging of the aircraft and to eliminate the interfered imaging of the infrared decoy meanwhile [1-4]. Hence the problem about the recognition and estimation of dynamic multi-object can be recognized and estimated by collaborative optimization algorithm.

Dynamic Multi-Objective Problem denotes that the optimization of objective function, the number of objective function and the dimension of decision space will change over time. It also includes the multi-objective problem of changing constraint conditions and parameters. Dynamic MultiObjective Problem is the combination of dynamic optimization problem and multi-objective optimization problem, which attracts a wider attention of domestic and overseas scholars [5-8]. In fact, Dynamic Multi-Objective Problem has a wide application background. For example, the multiobjective optimization of the missile's flying trace and the control parameter (angle of attack, elevation angle, etc.) should be processed as the target of a missile is changed. Affected by the severe weather, aircraft faults and the accidents that happen to the passengers on a flying aircraft, the factors such as the stranded time of the passengers and the cost of operation should be taken into account to get

*Address correspondence to this author at the College of Information Engineering, Jilin Business and Technology College, Changchun, Jilin, 130062, China; Tel: 13844198947; E-mail: livazl@126.com the aviation dispatcher re-optimized. The Dynamic MultiObjective Problem that widely exists in the physical world desires the appropriate algorithm to improve the ability to solve this problem.

There are a few research results about Dynamic MultiObjective Problem at present. Farina et al. create the first dynamic multi-objective function to put forward the algorithm of dynamic multi-objective optimization; Greeff et al. utilize the vector to estimate the particle swarm algorithm to solve the Dynamic Multi-Objective Problem with each object optimized by a particle. Enlightened by the recognition algorithm of the dynamic system mode, Talukder et al. put forward a new solution which uses a new operator to track the Pareto solution set of the decision space. ChiKiong et al. utilize the mutual collaboration of multiple populations to solve the Dynamic Multi-Objective Problem, which prove that multiple populations collaboration can fast track the changing Pareto optimal solution set and Pareto optimal front. Domestic scholars such as Liu Chunan et al. create a new pattern of dynamic multi-objective optimization algorithm and improve its convergence.

\section{THE PERFORMANCE CHARACTERISTICS OF INFRARED DECOY}

Infrared imaging can be taken as the effects acted by the factors such as time, space, and spectrum of radiation energy on propagation of the infrared radiation of scene in a medium. It can also be taken as a process of transferring and conversion of energy in detection equipment. As for the infrared detector, the primary task is to track and recognize the target aircraft. But the recognition degree of the target aircraft's tail flame and the infrared decoy is low for infrared detectors, which determines that the infrared imaging recognition and 
estimation give rise to the great difficulty and complexity in calculation. Thus, it has a great significance to analyze the dynamic characteristics of the infrared decoy and its trace estimation.

The infrared decoy is generally divided into rising time period, lasting time period and falling time period from emission to burning-up and disappearance. When the emission starts, the radiation intensity of burning will increase rapidly from 0 . And as the radiation intensity reaches the maximum extent, it begins to maintain the burning intensity to form the infrared image which is same as the attacking target aircraft tail flame. Along with the gradual exhaustion of the fuel, the radiation intensity decreases till it falls and burns up.

Cut off the aircraft, the infrared decoy will do parabolakind movement under the dual action of aerodynamic drag and gravity of projectile bodies. Above all, the drag acceleration to the infrared decoy is calculated as the following formula:

$\alpha_{d}(t)=\frac{\rho_{a} g v_{f}(t)^{2}}{2 \beta}=\frac{\rho_{a} g v_{f}(t)^{2} C_{d} A_{r e f}}{2 W(t)}$

In the above, is the atmospheric density; is the gravity acceleration; is the speed of the infrared decoy; $\beta$ is the trajectory coefficient; is the quality of the infrared decoy; is the resistance coefficient; is the referenced area of resistance coefficient.

$$
\left\{\begin{array}{l}
x_{f}(t+\Delta t)=x_{f}(t)+v_{f x}(t) \cdot \Delta t+\alpha_{d x} \cdot \Delta t^{2} / 2 \\
y_{f}(t+\Delta t)=y_{f}(t)+v_{f y}(t) \cdot \Delta t+\alpha_{d y} \cdot \Delta t^{2} / 2 \\
z_{f}(t+\Delta t)=z_{f}(t)+v_{f z}(t) \cdot \Delta t+\left(g+\alpha_{d z}\right) \cdot \Delta t^{2} / 2
\end{array}\right.
$$

Therefore, the infrared testing targets such as the infrared decoy and the tail flame of the target aircraft can be seen as particle swarms in the process of recognizing the infrared decoy. The motion trace of the infrared decoy is estimated after the multi-sample analysis of particle swarms and the combination of its own radiation characteristics and aerodynamic characteristics, which ultimately gives feedback to the detector to get a quick and exact estimation.

\section{DYNAMIC MULTI-OBJECTIVE PROBLEM AND ITS RELEVANT CONCEPTS}

Dynamic Multi-Objective Problem is demonstrated as the following formula:

$$
\min _{x \in X} f(x, t)=\left\{f_{1}(x, t), f_{2}(x, t), \mathrm{L} f_{m}(x, t),\right\}
$$

In the formula above, is the decision vector of the decision space $X$, is the target function set, is the number of the targets, $t$ is the function of time, $g, h$ is the corresponding inequality and the equality constraint function.

Some important definition of dynamic multi-objective optimization can be directly extended in the definition of the static multi-objective optimization.
Definition 1: Pareto dominance demotes that if

and,

$f_{1, j} \leq f_{2, j} \quad \forall j \in\{1,2, \mathrm{~L}, M\}$

Thus we can get: dominates $f_{2} \in F^{M}$, which can be demonstrated as $f_{1} \mathrm{p} f_{2}$.

Definition 2: the optimal front of dynamic Pareto

$P O F_{t}^{*}=\left\{f_{i, t}^{*} \mid / \exists f_{j, t}\right.$ p $\left.f_{i, t}^{*}, f_{j, t} \in F^{m}\right\}$

Definition 3: the optimal solution of dynamic Pareto.

$\operatorname{POS}_{t}^{*}=\left\{x_{i}^{*} \mid / \exists f\left(x_{j}, t\right)\right.$ p $\left.f\left(x_{i}^{*}, t\right), f\left(x_{j}, t\right) \in F^{m}\right\}$

The common point of dynamic multi-objective optimization and dynamic single-objective optimization is that the present optimal solution will change at the next moment. The key difference is that the former is a multi-dimensional target fitness space $F^{m}$. But the latter is just the single-objective optimization. Hence, the Pareto optimal front and Pareto optimal non-dominated solution set of Dynamic MultiObjective Problem can possibly change over time.

\section{HE DYNAMIC MULTI-OBJECTIVE RECOGNI- TION AND ESTIMATION ALGORITHM BASED ON PARTICLE SWARMS COLLABORATION}

At present, multiple population that utilizes various methods to collaborate in the framework of collaboration helps to improve the optimization efficiency of the algorithm. Potter illustrates in his doctoral dissertation in detail that the introduction of ecological models and the collaborative framework effectively improve the efficiency of classic evolutionary algorithm [9-12]. Major scholars apply the strategy of the paralleled search of multiple populations to respond to the changing environment. However, the multiple population parallel optimizations is not equal to the multiple population collaborative optimization, whose key difference is that the collaborative evolution has unique evolutionary dynamics mechanics, namely, multiple sub-population has mutual effects at individual level. And the multiple sub-populations become the selection pressure of each one of them and drive each other to improve the characteristics and complexity of each one to achieve the coevolution.

The multiple population collaborative optimizations is used to solve the complex problem, whose first step is to decompose the problem. Next is the collaboration of the population. The particle swarm collaborative model can be divided into two types according to the existing documents; one is based on space decomposing and the other is based on problem decomposing. As for the collaborative algorithm, it is not sufficient to apply the competitive mechanism and the collaborative mechanism. There in no competition between each particle swarm algorithm but the collaboration, because of the fact that the algorithm easily converges and gets into a local extremum. If the genetic algorithm has only selection 


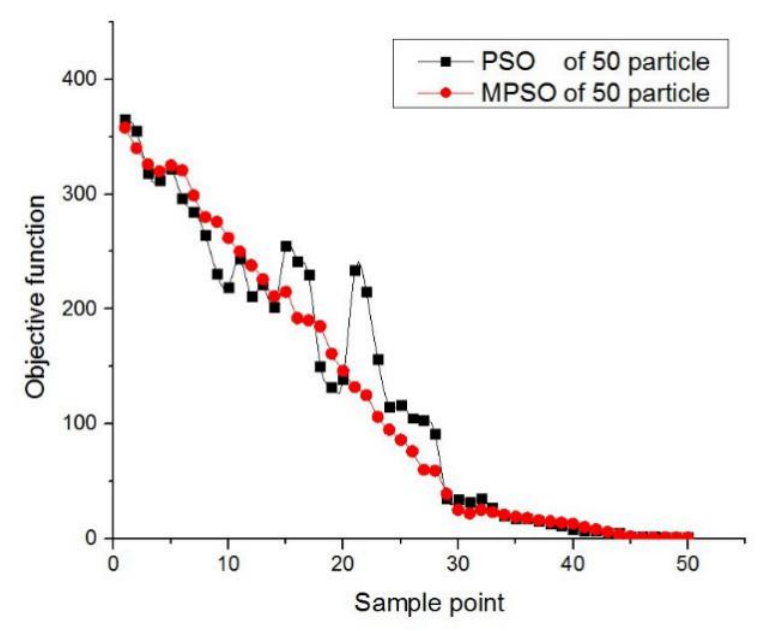

Fig. (1). The comparison diagram of PSO.

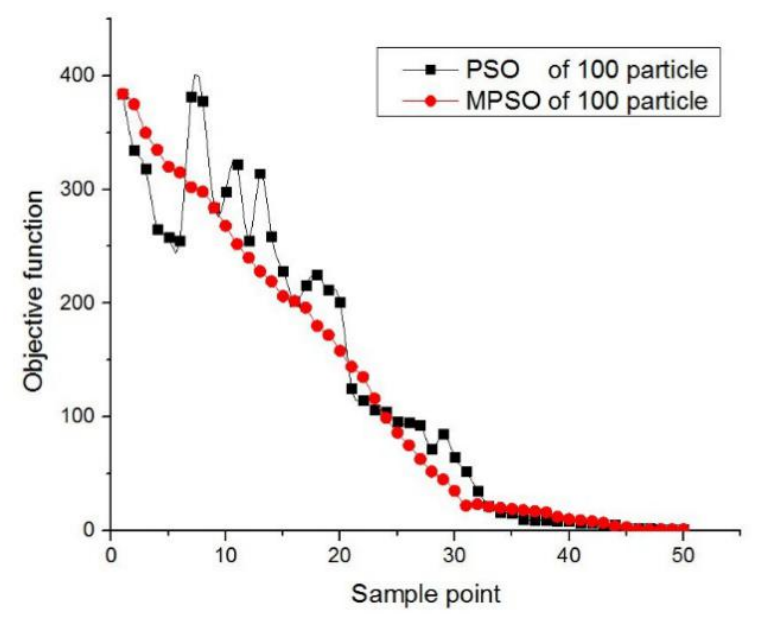

Fig. (2). The comparison diagram of PSO.

operator instead of mutation operator, the algorithm is easy to converge. Thus the search precision is greatly improved.

In the collaborative module, multiple sub-population is mainly applied to do the collaborative search in search space. The decomposition approach of the search space applied is display space decomposition, which means that each sub-population search on one dimension or multiple dimensions of the solution space. After that, multiple subpopulations collaborate to form a complete solution vector while calculating the individual fitness value. This kind of collaborative approach is proved effective in many documents, whose exact search on local area is adaptive. Based on the above notion, this paper introduces the multiple particle swarm collaborative optimization algorithm which utilizes the mixed competition and the collaborative mechanism of particle swarm algorithm when solving the dynamic multi-objective optimization problem.

\section{THE EXPERIMENTAL RESULTS}

\subsection{The Generation of Spread Spectrum Watermarking}

Firstly, PSO is initialized into a group of random particles (random solution). Next is to search for the optimal solution through iteration. During each times of iteration, a particle updates its own speed and location through individual extremum value and neighborhood extremum:

$$
\begin{aligned}
& v_{i}=w \times v_{i}+c_{1} \times f_{\text {rand }}\left(p_{i}-x_{i}\right)+c_{2} \times f_{\text {rand }}\left(l_{i}-x_{i}\right) \\
& x_{i}=x_{i}+v_{i}
\end{aligned}
$$

Among the above, inertia weight is used to control the effects on the present iteration speed which is acted by the speed of previous iteration. The global search features of the 


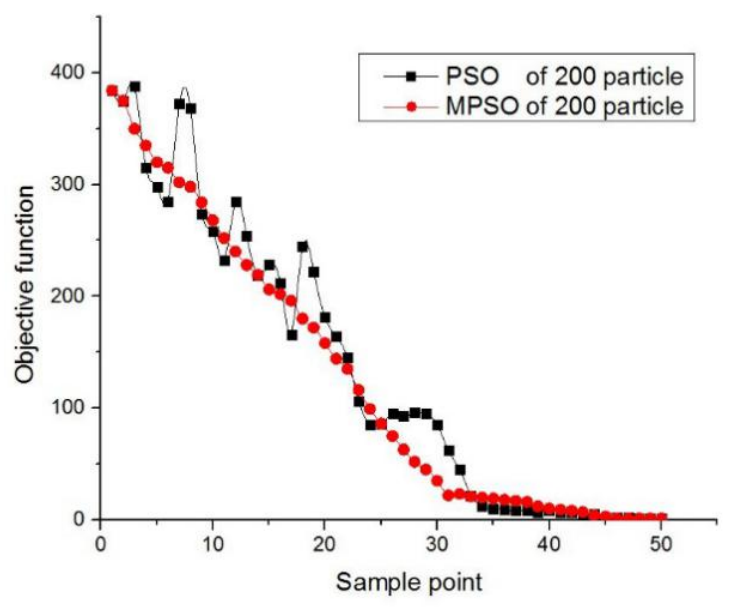

Fig. (3). The comparison diagram of PSO.

particle swarm optimization algorithm are realized by random initialization. The general inertia weight is $w \in[0,1]$. is the individual extremum and is the neighborhood extremum. is a random number from 0 to 1 . The formulas (1) and (2) are the speed displacement updating formulas which are the cores of the traditional PSO algorithm. In the formulas, this experiment sets up the learning factor: $c_{1}=c_{2}=2$. Suppose that the number of simultaneously releasing the infrared decoy is 9. Adding up with the target aircraft tail flame, the number of the object which is going to be recognized by the infrared seeker is 10 , which means that the space dimension where particles exist is $N=10$.

Initially, set 50 particles. Then make 10000 times iteration and circulate 100 times to get the optimal solution. During the process of 100 times search of optimization, ten of them find real attacking targets. The interference of infrared decoy is successfully excluded. To prove the effectiveness of the algorithm in this paper, the improved particle swarm algorithm put forward here is synthetically compared with the traditional particle swarm optimization algorithm. This experiment set three control groups which use the original particle swarm optimization algorithm, whose number of particles is 50, 100 and 200 respectively. Make sampling of optimal solution as each group completes relevant calculating amount and record the results. Then draw a comparison diagram as Figs. (1-3).

In this experiment, the changing curve of the optimization results has obvious convergence effects. And the speed of convergence is not affected by the changing number of particles. In the meantime, the above diagram demonstrates that the target function is greatly affected by particles when applying the original PSO, which is represented as: if the number of particles is too few, the effects of searching optimization are made poor though the speed of convergence is fast. It can be hardly ensured that the optimal solution can be found (50 particles and 100 particles); If the number of particles is high, the speed of convergence is slow though better solutions can be found (200 particles). But MPSO can well compromise the converging speed and the searching optimization to guarantee not only the converging speed but also better optimization results. In terms of the ultimate optimization results, the optimization result of improved algorithm is slightly better than that of the particle swarm algorithm.

\section{CONCLUSION}

This paper utilizes the Dynamic Multi-Objective Problem to recognize and estimate the detection image of the infrared imaging and to estimate and judge the trace of aerodynamic characteristics of the infrared decoy through the theory of multiple particle collaborative optimizations. This study designs the strategy of multiple particle collaborate repair and overcomes the shortcomings that the optimization results is greatly affected by the number of particles in standard algorithm. The strategy not only guarantees the optimization results but also the converging speed. This experiment demonstrates that the approach put forward in this paper can well solve the problem that the infrared seeker can recognize and estimate the inference of infrared decoy, which has great engineering significance.

\section{CONFLICT OF INTEREST}

The authors confirm that this article content has no conflict of interest.

\section{ACKNOWLEDGEMENTS}

Declared none.

\section{REFERENCES}

[1] K. Subbarao, and B. M. Shippey, "Hybrid genetic algorithm collocation method for trajectory optimization," Journal of Guidance, Control, and Dynamics, vol. 32, no. 4, pp. 1396 -1403, 2009.

[2] F. Bergh, and A.P. Engelbrecht, "Training product unit networks using cooperative particle swarm optimizers," In: Proceedings of International Joint Conference On Neural Networks, Washington. vol. 1, pp. 126-131, 2001.

[3] H. Yoshida, K. Kawata, and F. Yoshikazu, "A Particle swarm optimization for reactive power and voltage control considering voltage security assessment," IEEE Transactions on Power System, vol. 15 , no. 4, pp. 1232-1239, 2000.

[4] P. Williams, "Hermite-Legendre-Causs-Lobatto direct transcription in trajectory optimization," Journal of Gudance, Control and Dynamics, vol. 32, no. 4, pp. 1392-1395, 2009. 
[5] A. Wuerl, T. Crain, and E. Braden, "Genetic algorithm and calculus of variations based trajectory optimization technique," Journal of Spacecraft and Rockets, vol. 40, no. 6, pp. 882-888, 2003.

[6] M. Pontani, and B. A. Conway, "Particle swarm optimization applied to space trajectories," Journal of Guidance, Control, and Dynamics, vol. 33, no. 5, pp. 1429-1441, 2010.

[7] L. Gao, and H. B. Gao, "Particle swarm optimization based algorithm for cutting parameters selection," In: Proceedings of IEEE World Congress on Intelligent Control and Automation. Hangahou, 2004, vol. 4, pp. 284 7-2851.

[8] G. T. Huntington, and A. V. Rao, "Comparison of global and lo cal collocation methods for optimal control," Journal of Guidance, Control, and Dynamics, vol. 31, no. 2, pp. 432-436, 2008.

[9] J. S. Alsumait, J. K. Sykulski, and A. Al-Othman, "A hybrid GAPS-5QP method to solve power system valve-point economic dis- patch problems," Applied Energy, vol. 87, no. 5, pp. 1773-1781, 2010.

[10] K. E. Parsopoulos, and M. N. Vrahatis, "Recent approaches to global optimization problems through particle swarm optimization," Natural Computing, vol. 12, no.1, pp. 235-306, 2002.

[11] A. Lopez, C.A.C. Coello, A. Oyama, and K. Fujii, “An alternative preference relation to deal with many-objective optimization problems,” In: R.C. Purshouse, P.J. Fleming, C.M. Fonseca, S. Greco, J. Shaw, Eds. In: Proceedings of the $3^{\text {rd }}$ International Conference on Evolutionary Multi Criterion Optimization, Berlin, pp. 291-306, 2013.

[12] L.S. Batista, F. Campelo, F.G. Guimaraes, and J.A. Ramirez, "A comparison of dominance criteria in many-objective optimization problems," In: Proceedings of the IEEE Congress on Evolutionary Computation, Piscataway: IEEE Service Center, pp. 2359-2366, 2011.

Received: September 16, 2014

Revised: December 23, 2014

Accepted: December 31, 2014

(C) Zhai and Hu; Licensee Bentham Open.

This is an open access article licensed under the terms of the Creative Commons Attribution Non-Commercial License (http://creativecommons.org/licenses/by$\mathrm{nc} / 4.0 /$ ) which permits unrestricted, non-commercial use, distribution and reproduction in any medium, provided the work is properly cited. 